\title{
Más allá de las remesas. Transformaciones socioeconómicas y proyectos de migración y desarrollo en Ecuador
}

\author{
ANTONIO PALAZUELOS MANSO* \\ MARÍA DEL CARMEN VILLARREAL VILLAMAR**
}

RESUMEN: La visión predominante acerca del nexo existente entre migración y desarrollo considera la migración como un proceso capaz de generar y potenciar dinámicas de desarrollo en los contextos de origen de los migrantes. La evidencia empírica muestra, sin embargo, que las iniciativas de vinculación entre migración y desarrollo impulsadas por un gobierno pueden ser efectivas sólo si se acompañan de transformaciones socioeconómicas que permitan aprovechar los bienes y conocimientos de sus protagonistas. Independientemente de las modalidades de colaboración de los migrantes, las intenciones gubernamentales deben corresponderse con reformas de naturaleza social y económica que permitan su participación en la consecución de los objetivos de desarrollo estatales. Partiendo de este presupuesto el presente trabajo tiene por objetivo contrastar los objetivos y programas de la política migratoria ecuatoriana con las transformaciones socioeconómicas que están teniendo lugar en el país.

PALABRAS CLAVE: migración, desarrollo, política migratoria, reformas socioeconómicas, Ecuador.

ABSTRACT: The mainstream view on the nexus between migration and development considers migration as a process able to generate and reinforce development dynamics in the migrants' origin backgrounds. However, the empirical evidence shows that government initiatives linking migration and development could only be effective if they come together with socioeconomic transformations that would allow taking advantage of their beneficiaries' knowledge and goods. Regardless of the migrants' cooperation modalities, the government's intention should correspond with reforms of social and economic nature that would enable their participation in the achievement of the state's development goals. Starting from this assumption, this paper aims to contrast the goals and programmes of the ecuadorian migration policies with the socioeconomic transformations taking place in the country.

KEY WORDS: migration, development, migration policy, socioeconomic reforms, Ecuador.

\footnotetext{
* Profesor-investigador del Departamento de Economía Aplicada V de la Facultad de Ciencias Políticas y Sociología de la Universidad Complutense de Madrid, España.

** Doctoranda en Ciencias Políticas en la Universidad Complutense de Madrid, España.
} 


\section{Posturas en El Debate sobre migración y desarRollo}

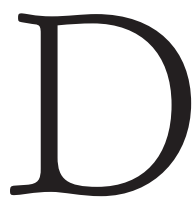

esde los inicios de la reflexión sobre el fenómeno migratorio, la variable desarrollo y su relación con la movilidad humana ha ocupado un lugar central en el debate y ha sido uno de los elementos más importantes a la hora de formular políticas migratorias, tanto en los países de origen como de destino de los migrantes (Mármora, 2002). En términos generales, la literatura sobre migración y desarrollo se divide entre visiones «pesimistas» $y$ «optimistas» que derivan, a su vez, de la formación de sus defensores y de la interpretación que estos últimos poseen sobre la migración. Así, mientras que mayoritariamente sociólogos y antropólogos se manifiestan escépticos frente a los beneficios de la migración, los economistas neoclásicos y los de la nueva economía de la migración defienden sus contribuciones al desarrollo en los contextos de origen (Portes, 2011).

Los pesimistas señalan, por ejemplo, los efectos negativos que los flujos migratorios producen en los países de origen en el ámbito demográfico, principalmente en lo que concierne al despoblamiento y envejecimiento de la población. Éstos cuestionan, además, la función de la migración como válvula de escape para la población desempleada y las potencialidades de las remesas, consideradas un elemento perjudicial para la economía, pues aumentan el consumo y no las inversiones productivas, generando dependencia y desestimulando la acción estatal para resolver los problemas de la población. Consideran asimismo el protagonismo asignado a los migrantes y las remesas como una forma de eludir las responsabilidades estatales e internacionales en la promoción del desarrollo (Castles y Delgado Wise, 2007; Carling, 2007; García Zamora, 2010, 2012).

$\mathrm{Al}$ analizar la migración calificada, los pesimistas adoptan generalmente una postura nacionalista, que en el ámbito económico coincide con posiciones intervencionistas y de regulación de la movilidad humana (Pellegrino y Calvo, 2001). Ven la migración de personas calificadas como un proceso que afecta negativamente a los países de origen, pues priva a estos últimos del capital humano y de la masa crítica necesaria para impulsar procesos de crecimiento y desarrollo económicos. Hablan asimismo de brain drain, considerando la 
migración calificada una pérdida neta para los países de origen y un obstáculo para la productividad de la población remanente (Brandi, 2006; Pellegrino y Calvo, 2001). Finalmente, los defensores de esta corriente cuestionan los beneficios de la migración de retorno aduciendo que no existen evidencias absolutas acerca de su aportación al desarrollo sino que ésta depende de una serie de factores como el contexto, las políticas impulsadas por el Estado de origen, el volumen y la tipología de la población retornada (Postelnicu, 2010).

Desde otro ángulo, los optimistas sobre la relación entre migración y desarrollo apoyan sus postulados en los efectos benéficos producidos en diversos ámbitos por las remesas, no sólo económicas sino también sociales. Señalan que la emigración permite reducir los problemas derivados del crecimiento demográfico y el desempleo, y que la perspectiva de emigrar puede generar algunos «efectos incentivo» de naturaleza positiva sobre la población local, como el aumento de la formación y habilidades de los trabajadores locales, además de los beneficios originados por la migración de retorno (Postelnicu, 2010). Desde el paradigma transnacional se evidencia además la potencialidad de las diásporas en los ámbitos económico, productivo y tecnológico, subrayando en este sentido el valor de los aprendizajes, contactos y experiencias acumuladas por estas comunidades (Abella y Ducanes, 2007; Portes, 2011).

Frente a la migración de personas calificadas, los optimistas tienen una perspectiva internacionalista y defienden la libre movilidad de las personas, por encima de intervenciones y regulaciones restrictivas (Pellegrino y Calvo, 2001). Hablan de brain gain, brain exchange, brain circulation y movilidad humana de talentos, considerando la salida de capital humano como un proceso que favorece la equilibrada distribución del talento y la producción a nivel mundial, beneficiando por lo tanto a todos los actores del proceso migratorio (Solimano, 2008).

Más allá de las posiciones en torno del tema, a la hora de realizar un cálculo objetivo de la relación existente entre migración y desarrollo, debería considerarse en primer lugar qué se entiende por desarrollo, así como los costes y beneficios que se producen en dicho proceso para los actores involucrados (Mármora, 2002). Asimismo, para calcular las posibles contribuciones de los 
migrantes a los procesos de desarrollo de sus países de origen deberían ser tenidas en cuenta las acciones promovidas por el gobierno para facilitar e impulsar tal proceso. La evidencia empírica muestra que la migración puede tener un impacto en los países en desarrollo siempre que en el contexto de origen exista «algo» donde los migrantes puedan regresar o iniciativas que les permitan colaborar y poner en práctica sus propias capacidades y experiencia (Portes, 2011). Dicho de otro modo, puede existir una relación virtuosa entre migración y desarrollo siempre que en el país de origen de los migrantes se den los primeros pasos para producir un cambio social, estructural y económico, sin el cual no puede haber desarrollo sostenido (Postelnicu, 2010).

\section{Migraciones internacionales en Ecuador}

Los desplazamientos internacionales de población en Ecuador tienen antecedentes en la movilidad interna asociada a los ciclos de crecimiento económico y urbanización del país, cuyos orígenes se remontan a mediados del XIX, y adquieren especial relevancia a partir de mediados del siglo xx (Acosta, 2006).

En el periodo 1951-1995, la génesis de los flujos migratorios fue la crisis económica del austro ecuatoriano, provocada por la disminución de la demanda de los tradicionales sombreros de paja toquilla, mejor conocidos como $\mathrm{Pa}$ nama Hats. Esta corriente migratoria de carácter irregular, mayoritariamente masculina y campesino-rural, se concentró territorialmente en los antiguos centros de comercialización del producto: Nueva York y Chicago (Herrera Carrillo y Torres, 2005).

En las décadas posteriores, la migración hacia Estados Unidos se mantuvo y se extendió a otras zonas del país como California, Minneapolis y Florida (Jokish, 2007). Paralelamente, debido a la atracción ejercida por el crecimiento económico de otros países se diversificó el perfil de los migrantes y se amplió el abanico de destinos, incluyendo países como Venezuela, Canadá y Colombia. Desde los noventa se registra también un flujo migratorio específico en la provincia de Loja con dirección a España, que posibilitaría más tarde la crea-

$110 \frac{\text { SEGUNDO SEMESTRE } 2013}{\text { MIGRACIÓN Y DESARROLLO NÚM. } 21}$ 
ción de las redes necesarias para el desplazamiento hacia este último país (Gómez Tornos y Colectivo IOÉ, 2007). ${ }^{1}$

Los patrones migratorios cambian radicalmente a partir de 1998 cuando tiene lugar un flujo migratorio sin precedentes en la historia de Ecuador que se extiende hasta 2004 y lleva a más de 700,000 personas a residir fuera del país. ${ }^{2}$ Calificada por algunos como boom, «fuga migratoria» o «estampida», esta nueva corriente fue el resultado de una serie de factores de naturaleza política, económica y social que determinó que el país experimentase la mayor crisis financiera de su historia y el empobrecimiento más acelerado registrado en la historia de América Latina en el periodo 1995-2000 (Acosta, 2006; Ramírez y Ramírez, 2005).

En esta segunda etapa se diversificó el perfil de los migrantes. Los flujos fueron prevalentemente femeninos y debido a los procesos de reunificación familiar fue significativa también la presencia de niños, jóvenes y ancianos. Éstos incluyeron además numerosas personas con formación secundaria y superior; y sin restar importancia a las causas económicas estuvieron también motivados por variables como la existencia de redes sociales y familiares; la búsqueda de oportunidades de mejora personal y familiar; y en el caso de la migración femenina por factores asociados a su condición de género como la violencia doméstica o problemas de naturaleza conyugal (Herrera, Carrillo y Torres, 2005; Ramírez y Ramírez, 2005; Camacho y Hernández, 2005).

Esta nueva corriente migratoria se caracterizó, asimismo, por la generalización del fenómeno migratorio a nivel nacional y por la diversificación de los destinos de los migrantes ecuatorianos que en respuesta a las políticas restrictivas aplicadas por Estados Unidos optaron por dirigirse hacia países con rela-

${ }^{1}$ Al ser la provincia de Loja una región fronteriza con Perú, esta nueva corriente, en opinión de algunos estudiosos, tendría como desencadenante las experiencias favorables de la emigración peruana de las zonas fronterizas, tradicionalmente dirigidas hacia España (Tornos et al.: 38, citado en Gómez et al., 2007: 33).

${ }^{2}$ Los cálculos basados en el «Primer Documento Oficial de Trabajo del Plan Nacional de Ecuatorianos en el Exterior» se refieren al periodo 1998-2003 y estiman un promedio de salida de 140,000 personas al año. Cabe recalcar, sin embargo, que estas cifras no consideraban los flujos de migración irregular, motivo por el que no daban cuenta de la real magnitud del fenómeno (ILDIS, 2004: 7-8). 
tivas facilidades en términos culturales, lingüísticos y de inserción como España e Italia, y en menor medida Francia, Bélgica, Suiza, Holanda, Alemania, Inglaterra, Chile y Argentina (Herrera, Carrillo y Torres, 2005; OIM, 2008).

En 2004, con la extensión de las políticas restrictivas al continente europeo y la aprobación del Acuerdo de Schengen entra en vigor la tercera fase de la migración ecuatoriana que se extiende hasta nuestros días. Resultado del fin de la mayor parte de los procesos de reunificación familiar y de los efectos de la crisis económica surgida en 2008, esta nueva etapa se caracteriza por la estabilización y desaceleración de los flujos. A diferencia de las corrientes precedentes, incluye también un proceso nuevo en el panorama migratorio ecuatoriano: el retorno de naturaleza voluntaria o forzada por parte de los migrantes, calculado en 176,775 personas, es decir, uno de cada cuatro emigrantes del periodo 2001-2010 (oIm, 2011: 49).

Actualmente, según los cálculos de la Secretaría Nacional del Migrante (Senami), se estima que existen entre 2 y 3 millones de ecuatorianos residiendo en el exterior. Debido a factores económicos, una coyuntura regional extremamente compleja y disposiciones aperturistas en materia migratoria, Ecuador es además territorio de destino, tránsito, refugio, migración circular, migración forzada, desplazamiento y retorno de miles de personas. Destacan a este respecto las más de 200,000 personas provenientes de países limítrofes como Colombia, Perú y Bolivia, junto a varios grupos de población originarios de Cuba, Haití, China, Pakistán y diversos países de África (OIM, 2008; OIM, 2012).

\section{PolítICA MIGRATORIA ECUATORIANA: CARACTERÍSTICAS Y OBJETIVOS}

Hasta finales de los años noventa y el inicio del boom migratorio ecuatoriano, los principales instrumentos en materia migratoria del país, la Ley de Migración y la Ley de Extranjería, databan de 1971 y estaban influenciados por la entonces vigente Doctrina de Seguridad Nacional que vinculaba la migración con la seguridad del Estado y priorizaba el control y la limitación de los flujos por encima de los derechos de los migrantes (Araujo y Eguiguren, 2009).

$112 \frac{\text { SEGUNDO SEMESTRE } 2013}{\text { MIGRACIÓN Y DESARROLLO NÚM. } 21}$ 
A partir de 1998, la magnitud de los flujos migratorios, la creciente importancia adquirida por las remesas y el contexto internacional favorable a la protección de los derechos de los migrantes, permitieron la entrada del fenómeno en la agenda pública y la conversión de la política migratoria en una política de Estado. Desde entonces la Constitución ecuatoriana reconoce el derecho a la doble nacionalidad y el derecho de voto de los migrantes, ${ }^{3}$ y aumentan de forma exponencial el número de proyectos de ley en materia migratoria, así como los mecanismos para combatir la trata y el tráfico de personas (Arízaga, 2005).

En 2000 se crea la Dirección General de Apoyo a los Ecuatorianos en el Exterior, y bajo el impulso del Ejecutivo, a partir de 2001 las acciones gubernamentales respecto al fenómeno migratorio adquieren un carácter de "política de Estado». En ese mismo año se aprueba el Plan Nacional de Migrantes Ecuatorianos en el Exterior, centrado en la protección del migrante por el Estado ecuatoriano, la atención a las familias de los migrantes en Ecuador y una relación más estrecha de Ecuador con los Estados de destino de la migración ecuatoriana que permitió la firma de acuerdos de regularización y de canje de deuda con el reino de España y la República italiana (Arízaga, 2005). Paralelamente, para impulsar el uso productivo de las remesas y la promoción del empleo en las comunidades de origen, se crea en 2002 el Programa de Ayuda, Ahorro e Inversión para los migrantes ecuatorianos y sus familias (Herrera y Moncayo, 2011).

En 2006, Rafael Correa Delgado, al frente del Movimiento Alianza País, se convierte en presidente de la República del Ecuador y partiendo de la definición de la migración ecuatoriana como una «tragedia nacional» y como «el mayor fracaso del modelo neoliberal», su gobierno impulsa una nueva política migratoria, inédita para la historia del país. ${ }^{5}$

${ }^{3}$ Derecho entrado en vigor mediante la reforma a la Ley de elecciones de 2002.

${ }^{4}$ En virtud de este proceso tuvo lugar en 2001 la firma con el reino de España del Acuerdo de «Regulación y Ordenación de los Flujos Migratorios», el «Programa de Conversión de Deuda» y el «Plan de retorno voluntario». En 2003 Ecuador e Italia suscribieron asimismo el «Acuerdo sobre la Conversión de Deuda en Proyectos de Desarrollo» (INECI, 2007).

${ }^{5}$ Discurso de posesión del presidente Rafael Correa el 15 de enero de 2007. 
De las reformas emprendidas por el nuevo gobierno destaca por importancia la creación mediante el Decreto Ejecutivo 150 de la Secretaría Nacional del Migrante (Senami) en 2007, y sucesivamente la aprobación de la actual Carta Magna del Ecuador en 2008 (Herrera y Moncayo, 2011: 22).

En primer lugar, la Senami es el órgano encargado de definir y ejecutar la política migratoria y está presente a nivel nacional así como en los principales destinos de la migración ecuatoriana ${ }^{6}$ (Herrera y Moncayo, 2011). Entre sus funciones actualmente se enumeran: a) el Programa de Posicionamiento de la Política Migratoria Integral del Ecuador con la relativa campaña internacional «Tod@s somos migrantes»; b) los servicios formativos, asistenciales y legales prestados a los migrantes y sus familias; c) el Plan «Bienvenidos a casa» que incluye asistencia e iniciativas productivas, laborales y de inversión para facilitar el retorno; y d) el Programa de Fortalecimiento Organizacional y Construcción de Redes Sociales en el hecho migratorio (Fores).

La segunda transformación cualitativa de la política migratoria ecuatoriana tuvo lugar con la aprobación en 2008 de la actual Constitución, redactada durante la Asamblea Constituyente de Montecristi y cuyo texto fue ampliamente conocido y debatido por la población, entre la que se incluyeron los migrantes, mediante los seis escaños asignados para este fin (Acosta, 2008).

Organizada bajo el principio rector del Buen Vivir o Sumak Kawsay la vigésima Carta Magna del Estado ecuatoriano constituye un hito en el reconocimiento de los derechos humanos de los migrantes, y desde la perspectiva de la movilidad humana analiza la migración en siete de sus nueve títulos, a lo largo de 58 artículos que evidencian la centralidad concedida al fenómeno.

${ }^{6}$ La Senami está presente en las ciudades más importantes de Ecuador a través de sus oficinas de atención. A nivel internacional se encuentra en los principales países de residencia de las comunidades ecuatorianas, teniendo oficinas de atención en Chicago, Santiago de Chile, Londres, Murcia, Barcelona, Génova y Roma. Esta institución cuenta además con casas ecuatorianas en las ciudades de Caracas, Nueva York, Madrid y Milán (Senami, 2013a). Pese a su importancia, según Quiloango (2011:30) la Senami no ha logrado resolver los problemas de superposición de competencias que dificultan la conducción de una política unívoca. En este sentido, además de la Senami, en el país existen otras instancias gubernamentales con atribuciones en materia migratoria entre las que sobresalen el Ministerio del Interior; el Ministerio de Relaciones Exteriores, Comercio e Integración; y el Ministerio de Relaciones Laborales.

$114 \frac{\text { SEGUNDO SEMESTRE } 2013}{\text { MIGRACIÓN Y DESARROLLO NÚM. } 21}$ 
Entre sus disposiciones se atribuye al Estado ecuatoriano la responsabilidad frente a la protección y salvaguardia de los derechos de los migrantes, la promoción de políticas públicas que beneficien al conjunto de la población y garanticen condiciones de permanencia, así como la posibilidad de un retorno digno de quienes salieron del país. Se garantizan a la par un conjunto de derechos civiles que van desde el reconocimiento del derecho al voto para los ciudadanos residentes en el exterior a la promoción de la participación mediante la presentación de propuestas y proyectos de ley. Bajo el principio de la ciudadanía universal, la Constitución establece como fines la búsqueda de la libre movilidad de todos los habitantes del planeta, el progresivo fin de la condición de extranjero y la exigencia del respeto de los derechos humanos de las personas migrantes (Benavides, 2008).

Partiendo de estas premisas, la actual política migratoria ecuatoriana, contenida en el Plan Nacional de Desarrollo Humano para las Migraciones 20072010, se compone de cinco objetivos prioritarios: 1. desarrollar en el Ecuador e impulsar en el mundo una política migratoria basada en el respeto y ejercicio de los derechos humanos, económicos, sociales y culturales de todas las personas; 2. generar y consolidar los vínculos de las personas migrantes con sus familiares y su país; 3. alentar la permanencia de los ecuatorianos en su país y construir las condiciones que hagan posible el retorno voluntario digno y sostenible de las personas emigrantes; 4. impulsar procesos de desarrollo humano para las personas migrantes, sus familiares y su entorno; y 5 . promover procesos de interculturalidad y de construcción de ciudadanía universal.

Fruto del diálogo entre instituciones del Estado y sociedad civil, el PNDHM 2007-2010 declara ser elaborado desde el «sur del mundo» con el objetivo de oponerse al enfoque predominante en materia migratoria que concibe esta última como un problema de seguridad. Fuertes críticas se realizan asimismo al excesivo énfasis puesto en las remesas como eje del desarrollo nacional, y al modelo neoliberal que determina que «se busquen trabajadores pero no personas». En oposición a este enfoque, el PNDHM define al migrante como «actor de desarrollo» y busca mediante mecanismos institucionales su vinculación y colaboración con el proceso de desarrollo del país. 
Desde su aprobación se han llevado a cabo una serie de iniciativas para salvaguardar los derechos de los migrantes que incluyen la apertura de nuevas sedes consulares, la firma de acuerdos de regularización de ciudadanos ecuatorianos en el exterior y el establecimiento de alianzas y acuerdos de cooperación en el ámbito económico, político y sanitario (Curtius, 2011: 177). Paralelamente, entidades como la Defensoría del Pueblo han emprendido acciones específicas de asistencia, orientación y defensa de los derechos de los migrantes en situaciones particulares como la crisis hipotecaria en España que ha afectado a muchos ciudadanos ecuatorianos (Senami, 2013a).

\section{Programas e iniciativas de Vinculación ENTRE MIGRACIÓN Y DESARROLLO EN ECUADOR}

Además de los programas y servicios asistenciales ofrecidos por la Senami para los migrantes ecuatorianos, existen una serie de iniciativas de vinculación, fomento de la inversión productiva y retorno. Entre éstas destacan por importancia las ofrecidas por a) el Plan Bienvenidos a Casa y las ofertas de la Banca del Migrante; $b$ ) el Plan de retorno para maestros y profesionales del Ecuador; c) el Plan de retorno «Ecuador Saludable Vuelvo por Ti» para profesionales de la salud; d) el Plan de concesión de tierras, y d) los Proyectos Yachay y Prometeo.

El Plan Bienvenidos a Casa, y dentro de éste el Fondo Concursable el Cucayo, prevé mediante un concurso la entrega de capital semilla no reembolsable a un migrante o grupo de migrantes retornados para el emprendimiento de negocios y proyectos productivos. El proyecto concebido como una inversión de riesgo compartido, además del capital semilla, implica la entrega por los beneficiados de una contraparte. Éstos se benefician además de capacitación, apoyo y asistencia técnica para sus emprendimientos que suelen ser micro y medianas empresas (Herrera y Moncayo, 2011: 43-44). Hasta finales de 2012, según datos de la Senami, 11,870 personas migrantes se han beneficiado de esta iniciativa mediante la capacitación para el emprendimiento de negocios (Senami, 2013b). 
De forma semejante la Banca del Migrante ofrece a través de las operadoras de la Corporación de Finanzas Popularesy Solidarias del Ecuador (Conafips), créditos por un máximo de 20 mil dólares a los migrantes y sus familias para financiar actividades productivas, de comercio y servicios y de ampliación y modelación de las viviendas. Por medio de esta entidad hasta finales de 2012 habían sido concedidos 30'199,768 créditos (Senami, 2013a).

Además de la concesión de créditos y capacitación técnica existen varios programas de retorno organizado para profesionales de la educación y de la salud. Así por ejemplo, el Plan de Retorno para Maestros prevé la participación de docentes ecuatorianos residentes en el exterior en los concursos de mérito y oposición organizados por el magisterio del país. Dicho Plan, organizado por el Ministerio de Educación en coordinación con la Senami, tiene por objetivo el retorno organizado de profesionales del sector a través de un contrato temporal de trabajo que permita su reinserción en la carrera docente. Con objetivos semejantes el Plan Ecuador Saludable Vuelvo por Ti, coordinado por el Ministerio de Salud Pública del Ecuador y la Senami, prevé cubrir las necesidades técnicas y operativas de la red pública nacional de salud por medio de la contratación de profesionales ecuatorianos del sector residentes en el exterior, o residentes en el país por un periodo limitado después de un proceso migratorio.

Dentro de las iniciativas para fomentar el retorno organizado y productivo de los migrantes destaca además el Plan Tierras coordinado por el Ministerio de Agricultura, Ganadería y Pesca (Magap) y la Senami que contempla la entrega de títulos de propiedad de 3 mil hectáreas de tierra en las provincias de Guayas y Manabí a cooperativas con fines productivos constituidas por migrantes, específicamente pequeños y medianos productores que emigraron a España ${ }^{7}$ (Senami, 2013a).

En paralelo, existe además un conjunto de iniciativas que tienen por objetivo el apoyo a los profesionales ecuatorianos residentes en el exterior ${ }^{8}$ y las

${ }^{7}$ Cabe recalcar que hasta la fecha el Plan de Retorno de Docente y el Plan Tierras han sido ejecutados exclusivamente en España.

${ }^{8}$ Entre éstas se incluyen la participación de los migrantes en los programas nacionales de becas y ayudas para la realización de estudios, y la financiación de proyectos artísticos. 
iniciativas Yachay y Prometo que si bien no han sido concebidas exclusivamente para los migrantes, pretenden fomentar el retorno de profesionales ecuatorianos con altos niveles de formación.

El Proyecto Yachay, por ejemplo, también denominado Ciudad del Conocimiento, tiene como eje central el conocimiento y la transformación de la matriz productiva ecuatoriana, tradicionalmente basada en la exportación de bienes primarios. El proyecto, inspirado en la ciudad japonesa de Tsukuba, ha sido concebido como «la primera ciudad planificada del Ecuador» $\mathrm{y}$ «la primera ciudad tecnológica de América Latina». Entre sus objetivos prevé el establecimiento de una universidad experimental, Zonas Especiales de Desarrollo Económico (ZEDE) e instituciones de investigación públicas y privadas que generen conocimientos y tecnologías en el país en ámbitos como las ciencias de la vida, las tecnologías de la información y comunicación (TICs), la petroquímica, la nanociencia, la energía renovable y el cambio climático (Yachay, 2013).

Dentro de la misma estrategia, el Programa Prometeo busca fortalecer las capacidades de investigación de las instituciones científicas del país por medio de la vinculación de científicos de reconocida trayectoria, también denominados «viejos sabios». Entre sus objetivos se calcula la llegada al país de más de 100 especialistas para trabajar en institutos nacionales de investigación, universidades, escuelas politécnicas y diversas entidades del sector público y privado que producen ciencia en Ecuador. Su objetivo es la vinculación de reconocidas personalidades del ámbito científico a instituciones ecuatorianas por medio de estancias temporales y colaboración permanente en el desarrollo de proyectos de investigación conjuntos. Dentro de las áreas de colaboración, este proyecto contempla varias posibilidades, pero prioriza especialmente disciplinas relacionadas con las ciencias de la vida, los recursos naturales y la producción e innovación (Senescyt, 2013b).

Tras este breve repaso al proceso migratorio ecuatoriano y los principales elementos de las políticas públicas respecto a la migración, vamos a referirnos -como planteábamos en la introducción- a la transformación productiva que se está produciendo en el país con el fin de acercarnos a una primera evalua-

$118 \frac{\text { SEGUNDO SEMESTRE } 2013}{\text { MIGRACIÓN Y DESARROLLO NÚM. } 21}$ 
ción sobre la coherencia entre los objetivos y los programas de política migratoria en relación con la realidad productiva y social del país.

\section{TRANSFORMACIÓN PRODUCTIVA DE «la Revolución Ciudadana»?}

En el Plan Nacional para el Buen Vivir (PNDBV) se recoge la nueva estrategia de desarrollo que se pretende implantar en el país, de manera que posibilite un nuevo modelo de acumulación y (re)distribución que supere la estrategia de crecimiento primario-exportadora recuperada por el neoliberalismo, donde el objetivo de la transformación productiva estructural se visualiza como la forma de aprovechar las ventajas del país, definidas en torno de la biodiversidad, la petroquímica, la bioenergía y los servicios ambientales, que sean compatibles en el largo plazo con la sostenibilidad ambiental y con las mejoras de las condiciones de vida de la población (Senplades, 2009).

En la primera fase o etapa (2009-2013), considerada de transición, se entiende que el crecimiento económico estará todavía sustentado sobre la base primario-exportadora, pero deben darse pasos significativos en la sustitución selectiva de exportaciones con el impulso — a través de la inversión pública-, de sectores que generen valor y actividades que garanticen el empleo y la supervivencia digna de la población, como el turismo o la agroindustria, con un fuerte sustento en la economía popular y solidaria.

Según uno de los documentos básicos elaborados desde el gobierno, la Agenda para la transformación productiva (2011) se realizaría sobre la base de ocho pilares ${ }^{10}$ que permitirá cambiar el patrón de especialización productiva

9 Véase Palazuelos (2013) y Palazuelos y Villarreal (2012).

${ }^{10}$ Los ocho pilares serían: 1) el cambio en la matriz productiva y en la especialización productiva y exportadora, 2) la reducción de la heterogeneidad estructural, 3) la democratización de los recursos, 4) el empleo de calidad, 5) el talento humano y el sistema integral de innovación y emprendimiento, 6) la productividad y competitividad sistémica, 7) el crecimiento verde, es decir, la sostenibilidad ambiental, y 8) el cambio cultural e imagen del país (Ministerio de Coordinación de la Producción, Empleo y Competitividad, 2011: 73-94). 
consolidando un modelo que fundamente el desarrollo económico en el conocimiento como generador de valor agregado, apoyando la producción y exportación de los 14 sectores considerados prioritarios y las 5 industrias consideradas estratégicas ${ }^{11}$ para el cambio de la matriz productiva.

El cambio de la matriz productiva significaría tanto potenciar y fortalecer los sectores productivos donde ya se tenga ventajas comparativas (por ejemplo, en la agroindustria a través de la incorporación del conocimiento científico y tecnológico), como apoyar el desarrollo de los sectores priorizados y las industrias estratégicas de manera que se permita la generación de mayor valor agregado en la producción y las exportaciones. Esto supone un enorme desafío ya que exigirá cambios profundos en las estructuras económicas e institucionales del país. En este sentido, la Senplades (2013) se encontraría trabajando en una doble vía:

a) La sustitución selectiva de importaciones, de manera que la producción local vaya desplazando progresivamente los bienes importados, bien sobre la base de ampliar la escala productiva nacional o la generación de nueva producción, lo que implica configurar políticas productivas estructurales intersectoriales que fortalezcan la competitividad sistémica. En esta línea estaría la intensa política de inversión en obra pública (construcción y reparación de carreteras, puertos y aeropuertos) o la Ley Orgánica de Educación Superior (que facilitaría la producción de conocimiento y el desarrollo de ciencia y tecnología).

b) La diversificación y promoción de exportaciones orientada a mejorar la calidad y competitividad de los productos no tradicionales que incorporen valor agregado para su colocación en los mercados internacionales.

${ }^{11}$ Los 14 sectores considerados prioritarios son: 1) turismo, 2) alimentos frescos y procesados, 3) energías renovables, 4) productos farmacéuticos y químicos, 5) biotecnología: bioquímicos y biomedicina, 6) servicios ambientales, 7) metalmecánica, 8) tecnología: hardware y software, 9) plásticos y caucho sintético, 10) confecciones, ropa y calzado, 11) vehículos automotores, carrocerías y partes, 12) transporte y logística, 13) construcción, y 14) cadena forestal sustentable y productos madereros procesados. Mientras que las industrias estratégicas serían: 1) refinerías, 2) astilleros, 3) petroquímica, 4) metalurgia y 5) siderúrgica (Ministerio de Coordinación de la Producción, Empleo y Competitividad, 2011).

$120 \frac{\text { SEGUNDO SEMESTRE } 2013}{\text { MIGRACIÓN Y DESARROLLO NÚM. } 21}$ 
Uno de los instrumentos esenciales para la consecución de la transformación productiva consistiría en reducir la heterogeneidad estructural y las brechas de productividad, externas e internas, que el modelo neoliberal habría agudizado, potenciando una profunda concentración: a) productiva, b) empresarial, c) escasa oferta de productos y servicios, mayoritariamente primarios y con escaso valor agregado, $\mathrm{y} d$ ) de mercados de destino en el comercio internacional (Ministerio de Coordinación de la Producción, Empleo y Competitividad, 2011: 30-54).

La denominada brecha externa ${ }^{12}$ se caracterizaba con la asimetría en las capacidades tecnológicas con respecto a la brecha internacional y la concentración del comercio, mientras que la brecha interna lo hacía a través de la notable diferencia de productividad entre sectores productivos y al interior de los mismos. ${ }^{13}$

La fuerte heterogeneidad productiva tenía su expresión en una profunda concentración empresarial, territorial y social. La brecha empresarial se expresa a partir de considerar que las grandes empresas del país concentran $78 \%$ de las ventas, mientras que las micro y pequeñas empresas (que suponen $94 \%$ del total), apenas representan $7 \%$ del total de las ventas, aunque generen $42 \%$ del empleo remunerado (generalmente de escasa calificación, bajos salarios y nulas prestaciones), mientras que las grandes empresas (que

12 Según el Ministerio de Coordinación de la Producción, Empleo y Competitividad (2011: 32-37), los indicadores convencionales que expresan los niveles tecnológicos eran claramente negativos para Ecuador en 2008, así el gasto público en educación apenas llegaba a 1\% del PIB (el más bajo de América Latina), mientras que el gasto en I+D expresado en infraestructura educativa era enormemente bajo. Por su parte, la concentración del comercio quedaba en evidencia al considerar que a pesar de exportar un total 3,107 productos, apenas cinco de ellos representaban 74\% del total de exportaciones ( $89 \%$ en 10 productos) y solamente dos países-regiones (Estados Unidos y Unión Europea) recibían 60\% de las exportaciones (10 destinos el 80\% del total).

${ }^{13}$ Apenas seis sectores económicos representan 65\% del PIB total, mientras que los sectores que generan poco valor agregado son los que mayor empleo concentran (58\%), siendo generalmente un empleo poco calificado y con salarios muy bajos (el salario medio de las microempresas es $60 \%$ de las grandes). Esto llevaba a que la productividad laboral media de las grandes empresas sea el doble que el de las microempresas (Ministerio de Coordinación de la Producción, Empleo y Competitividad, 2011: 38-44). 
apenas son $6 \%$ del total), captan $58 \%$ del empleo remunerado. ${ }^{14}$ Por su parte, a nivel territorial, la concentración también es enorme ya que tres regiones (Guayas, Pichincha y Azuay, donde se encuentran las tres grandes ciudades del país: Guayaquil, Quito y Cuenca) concentran 86\% del total de empresas, esencialmente las de mayor tamaño, donde se encuentran los mayores niveles de inversión y tecnología, y los principales sectores y ramas productivas más dinámicas.

Este modelo productivo fuertemente concentrador lleva inexorablemente a una brecha social de grandes dimensiones, que se expresaba en 2006 en la concentración del ingreso, donde $10 \%$ más rico poseía $42 \%$ de la riqueza (y 20\%, 58\% del total), mientras que 10\% más pobre apenas «arañaba» $1.1 \%$ de la riqueza.

Para tratar de transformar esta realidad productiva la Agenda ha desarrollado numerosas acciones en muy diversos ámbitos tratando, a partir de diversos planes productivos «llegar a consolidar una estrategia de diversificación productiva y territorial que permita avanzar realmente en el cambio de la matriz productiva». No obstante, y a pesar de los favorables resultados obtenidos ${ }^{15}$ en términos de crecimiento industrial, mayor diversificación exportadora, enormes esfuerzos inversores en infraestructuras y mejoras laborales y sociales derivadas de ellos, los cambios en la matriz productiva

14 Según el Ministerio de Coordinación de la Producción, Empleo y Competitividad (2011: 45-54), otra expresión de esta alta concentración empresarial se expresa a través del capital accionarial, ya que $92 \%$ del total del capital accionariado del país se concentra en los cinco mayores accionistas, poseyendo el mayor de ellos $72 \%$ del total.

${ }^{15}$ Los datos facilitados por el gobierno evidencian el fuerte crecimiento experimentado por el sector no petrolero de la economía en algunos años (8.8\% en 2011), el mayor dinamismo de las exportaciones no petroleras (crecen un $20 \%$ en 2011), habiéndose diversificado significativamente los mercados de exportación, las fuertes inversiones públicas realizadas para el fomento productivo (9,380 millones de dólares entre 2007-2011) lo cual ha permitido el financiamiento de 165 proyectos productivos, así como elevar la capacitación especializada y mejoras importantes en las infraestructuras $(8,884 \mathrm{~km}$ de carreteras, 78 puentes, cinco aeropuertos, etcétera), que ha posibilitado mejorar los niveles de empleo de mayor calidad, el acceso de un mayor número al Instituto Estatal de Seguridad Social (IESS) (59.8\% de afiliados) y mejorar los ingresos de las familias; así, a mediados de 2012 el ingreso mensual cubría 93\% de la canasta básica (Ministerio de Coordinación de la Producción, Empleo y Competitividad, 2012).

$122 \frac{\text { SEGUNDO SEMESTRE } 2013}{\text { MIGRACIÓN Y DESARROLLO NÚM. } 21}$ 
todavía no son evidentes en los grandes agregados y se podría decir que la transformación productiva resulta ser el obstáculo más difícil de superar dentro del proyecto gubernamental y nacional.

\section{TransFormación PRODUCTIVA PARA EL DESARROLlo de ECUAdOR E INSERCIÓN LABORAL DE LOS MIGRANTES}

Un análisis de los grandes agregados sectoriales y ramales de la estructura productiva pareciera evidenciar escasos cambios en la matriz productiva, lo cual podría ser explicable por diversas razones. ${ }^{16}$ No obstante, si tenemos en consideración los principales componentes productivos (del PIB) y las grandes líneas del comercio exterior ecuatoriano encontramos —en relación con los grandes objetivos de corto plazo propuestos- que:

1. Es posible que las importantes inversiones públicas realizadas —que han posibilitado llevar a cabo proyectos productivos y mejoras en las infraestructuras físicas del país - expliquen, en parte, el significativo crecimiento de

${ }^{16}$ La más evidente podría ser el escaso margen de tiempo del proceso, dado que el PNDBV se edita en 2009, y su desarrollo a partir de políticas productivas y comerciales dirigidas a cumplir los objetivos de corto y medio plazo se comienzan a aplicar a partir de 2010, lo cual evidenciaría los resultados de apenas dos o tres años, tiempo insuficiente para producir grandes cambios estructurales. Por otro lado, es indudable que sería necesario realizar un análisis mucho más desagregado, microeconómico, en una triple dimensión: ramal, empresarial y regional, con el fin de poder evaluar realmente los resultados de los nuevos fenómenos productivos al calor de las políticas públicas de inversión selectiva, nueva reglamentación pública e iniciativas de inversión y producción tanto privadas como públicas, además de los proyectos colectivos y sociales llevados a cabo en este sentido, siendo de especial interés los avances conseguidos por la economía popular y solidaria. Por último, un análisis más desagregado de los resultados del comercio exterior, de exportaciones e importaciones a nivel micro, trataría de evidenciar los posibles cambios en la estructura de dicho comercio, y las razones productivas-comerciales de dichos cambios, además del análisis de los principales agentes que llevan a cambio dicha actividad. Aunque en estos momentos nos encontramos comprometidos con dicho análisis más riguroso, las dificultades de acceso estadístico y el tiempo nos impiden de momento elaborar conclusiones sobre dicho proceso y trasladamos los posibles resultados a próximas publicaciones. 
ciertos sectores y ramas productivas, ${ }^{17}$ que estarían reflejando el cambio productivo al que se aspira. No obstante, el escaso peso relativo de algunos de los más dinámicos en el conjunto de la producción nacional, hace que aun aportando una proporción importante al crecimiento económico, impida tener mayores efectos arrastre en el conjunto productivo y el empleo.

2. Estos pequeños cambios productivos no se reflejan decididamente en los parámetros de especialización e inserción comercial internacional, en la medida que no se aprecian cambios significativos (aunque sí algunos elementos transformadores a señalar) en la estructura de exportación e importación que reflejaran una «nueva forma» de inserción internacional. La estructura exportadora apenas expresa pequeños cambios, dado el peso preponderante del petróleo crudo (en torno de $58 \%$ del total), los productos tradicionales (que incluso han elevado su importancia relativa en los últimos años) y las ramas manufactureras tradicionales dentro de la exportaciones no-tradicionales, aunque se aprecia un crecimiento significativo en productos como camarón, atún, pescado y enlatados de pescado (en conjunto, $17.8 \%$ en $2011)$, vehículos y otras manufacturas del metal ( $8.9 \%$ ) o productos mineros (4.4\%); aunque quizás el cambio más significativo se produce en el destino de los flujos de exportación, debido a la pérdida de peso de Estados Unidos (se pasa de $53.6 \%$ en 2006 a 43.8\% en 2011), y el creciente protagonismo de la Unión Europea (13\% en 2011) y los países latinoamericanos de ALADI (24.4\%), con un peso significativo de las exportaciones hacia Chile, Perú, Colombia, Venezuela y Panamá, y novedoso (aunque todavía poco significativo), las exportaciones hacia otras regiones como Asia (con 4\% en 2001, y una participación alta de Japón, China) o Rusia (con 3.1\%).

3. Igualmente, tampoco se aprecian cambios significativos en el perfil de las importaciones, tanto en sus componentes como en el origen de los flujos, aunque pudiera considerarse una incipiente sustitución de importaciones la reducción relativa de bienes de consumo (esencialmente no duraderos) y

17 Por ejemplo, en 2011, los sectores que reflejan mayor crecimiento serán suministro de electricidad y agua (29.5\%), acuicultura y camarón (22.1\%), construcción (21.6\%), con crecimiento algo menores aparecen sectores como alojamientos (13.4\%), refino de petróleo $(12.8 \%)$, comunicaciones $(12.5 \%)$, servicios financieros $(11.4 \%)$, mientras que una gran número de sectores: gobierno, transporte, comercial, actividades profesionales y técnicas, pesca, agrario y manufacturas, tienen crecimiento igual o superiores a 5\% (BCE, 2012).

$124 \frac{\text { SEGUNDO SEMESTRE } 2013}{\text { MIGRACIÓN Y DESARROLLO NÚM. } 21}$ 
materias primas, aunque el incremento significativo de las importaciones de combustibles y lubricantes (que pasan de representar $16.6 \%$ en 2006 a $22.7 \%$ en 2001), y el aumento de precios, distorsionan los términos relativos del conjunto. ${ }^{18}$ En cuanto al origen de las importaciones, no hay cambios significativos destacando, en 2011, las provenientes de Estados Unidos (25.2\%), ALADI (30.5\%, con un peso importante de países como Brasil, Perú, Colombia y Venezuela) y la Unión Europea (10\%).

Por lo tanto, esta mejora productiva, aun permitiendo ciertos avances en relación con la diversificación de los componentes exportadores y la dirección de los flujos, parecieran insuficientes para conseguir de manera significativa el objetivo de sustitución selectiva de importaciones y exportaciones que representa el principal reto de esta primera etapa del proceso transformador.

Con referencia a la incidencia de estas transformaciones productivas y exportadoras a la generación de empleo y la mejora de su calidad, si nos atenemos a las principales variables que explican el comportamiento del mercado de trabajo en Ecuador durante el periodo 2007-2012, los principales resultados serían: ${ }^{19}$

1. En relación con la generación de empleo, se aprecia en la totalidad del periodo - dado el deterioro de los años de incidencia de la crisis internacional- una escasa mejora relativa, que se evidencia en un incremento de la tasa de ocupación (pese a la reducción de la tasa de participación), y una reducción de la tasa de desempleo urbano, aunque éste sigue siendo importante para los jóvenes. ${ }^{20}$

${ }^{18}$ Los bienes de consumo pasan de 22\% en 2006 a 20.1\% en 2011 (los no duraderos lo hacen de $13.4 \%$ a $11.7 \%$ ) del total de importaciones, mientras que las materias primas pasan del $33.2 \%$ a $30.4 \%$ (esencialmente de materias primas para la industria, que pasa de $25.2 \%$ a $22.6 \%$ ), y los bienes de capital de $27.9 \%$ a $26.7 \%$, para los mismos años.

${ }^{19}$ A la hora de utilizar los datos referidos al mercado de trabajo hay que tener en consideración las diferentes fuentes (ENUDE, BCE, OIT) que utilizamos, así como la base estadística de partida (la PET a partir de los 10 o los 15 años, dado que obtenemos resultados relativamente diferentes.

${ }^{20} \mathrm{La}$ tasa de desempleo urbano se reduce de $8.1 \%$ en 2006 a $4.9 \%$ promedio de los tres primeros trimestres de 2012 , siendo de $4.4 \%$ para los hombres, $5.6 \%$ para las mujeres y $13.4 \%$ para los jóvenes entre 15 y 24 años, para 2012 (oIT, 2013). 
2. La generación de empleo se ha producido tanto en el trabajo asalariado (aunque preferentemente en establecimientos de cinco o menos trabajadores), como en el trabajo no asalariado (también esencialmente en relación con los patronos de establecimientos de cinco o menos trabajadores, y trabajadores independientes no profesionales, técnicos o administrativos), además de empleos en el servicio doméstico. A nivel sectorial se aprecian escasos cambios importantes, con un incremento relativo del empleo en los sectores de transporte, almacenamiento y comunicaciones y establecimiento financieros (con poco peso en términos absolutos), mientras que se mantienen estables o ligeramente a la baja en los sectores mayormente empleadores: comercio (34.2\%); servicios comunales, sociales y personales $(21.4 \%)$ e industria $(13.2 \%)$, manteniéndose el fuerte sesgo de género a nivel sectorial (el empleo femenino se concentra casi en apenas dos sectores, comercio (42.9\%) y servicios comunales, sociales y personales (30.8\%) (orT, 2013).

3. Donde sí se aprecia cambios significativos es en la calidad del empleo si nos referimos — como hace la orT a través del «trabajo decente» — a la cobertura de previsión social en Salud o Pensiones donde se ha elevado el porcentaje de trabajadores cubiertos de $33.6 \%$ a $47.3 \%$, pero con mejoras de importancia esencialmente en los trabajadores asalariados y en menor medida en los no asalariados y servicio doméstico. No obstante, todavía existen grandes colectivos sin acceso a estas prestaciones: $25.5 \%$ de los trabajadores asalariados que laboran en establecimientos de cinco o menos trabajadores, $23.8 \%$ del total de los no asalariados, y de ellos, tan sólo 19\% de los trabajadores independientes (OIT, 2013).

4. Sin embargo, la tasa de ocupados plenos apenas alcanza a 52.1\% en las áreas urbanas y tan sólo $22.7 \%$ en el área rural, mientras que la tasa de subocupación se eleva a $39.8 \%$ en el área urbana y nada menos que a $74.6 \%$ en la rural. ${ }^{21}$

${ }^{21}$ Datos facilitados por el BCE (2013) considerando la PET a la población de 10 y más años, donde se aprecian ciertas mejoras (se pasa de 43.6\% en 2007 a $52.1 \%$ en 2012 en ocupados plenos en áreas urbanas y de $19.9 \%$ a $22.7 \%$ en rurales, mientras que la subocupación se reduce de $48.8 \%$ a $39.8 \%$ en áreas urbanas y de $77 \%$ a $74.6 \%$ en rurales), aunque insuficientes. También sería destacable el hecho de que las mejoras no sólo abarcan al mundo rural, sino al conjunto de las provincias, ya que mayoritariamente se ha mejorado el empleo, aunque sigue habiendo diferencias regionales importantes.

$126 \frac{\text { SEGUNDO SEMESTRE } 2013}{\text { MIGRACIÓN Y DESARROLLO NÚM. } 21}$ 
Por ello, entendemos que los avances conseguidos en la generación y mejora del empleo parecieran no recoger, todavía, los posibles frutos de los avances conseguidos en la transformación productiva y la mayor diversificación y competitividad en sectores de mayor valor agregado, si nos atenemos a los datos descritos y al hecho de que los establecimientos (empresas) de seis o más trabajadores han perdido peso relativo, al igual que los trabajadores independientes, profesionales y administrativos, aunque se hayan elevado sectorialmente algunas de las actividades que demandan personal de mayor calificación.

Por último, y a manera de una especie de breve conclusión de este trabajo - en la línea de los objetivos marcados-, podemos afirmar que las iniciativas de vinculación entre migración y desarrollo establecidas por el gobierno ecuatoriano han estado acompañadas de reformas socioeconómicas que tienen como objetivo modificar la matriz productiva del país y mejorar efectivamente el bienestar de la población. Entendemos que plantear una respuesta concluyente sobre la medida de la incidencia de la transformación productiva, los cambios en las condicionales laborales del país y las políticas públicas migratorias, en relación con las posibilidades reales de inserción de la migración de retorno es algo precipitado, no obstante, podemos sostener la hipótesis provisional de que la inserción de los migrados al mercado de trabajo ecuatoriano no está siendo todo lo satisfactoria que se deseara si nos atenemos a criterios generales, aunque es difícil discernir — salvo que se realicen análisis micro a nivel sectorial, empresarial o regional-, dadas las distintas situaciones laborales (formación, capacidades, aprendizajes) del grupo de ecuatorianos retornados, que exigen un análisis de mayor profundidad y concreción que el abarcado en este texto.

\section{Biblografía}

ABeLla, Manolo y Ducanes Jeffrey (2007), «żEs el transnacionalismo un nuevo paradigma para el desarrollo?», en Castles Stephen y Delgado Wise Raúl (coords.), Migración y desarrollo: Perspectivas desde el Sur, México, Miguel Ángel Porrúa, Universidad Autónoma de Zacatecas. 
Acosta, Alberto (2008), «La compleja tarea de construir democráticamente una sociedad democrática», La Tendencia no. 8.

(2006), La migración en el Ecuador: oportunidades y amenazas, Quito, Corporación Editora Nacional.

Araujo, Lorena y Eguiguren María Mercedes (2009), «Diagnóstico de la normativa en materia migratoria en la región andina y propuesta técnica de fortalecimiento de la exigibilidad de los derechos de los migrantes andinos», en http://www. redandinademigraciones.org/documentos/cat_view/41-doctos-red-andina (22/09/2012).

ARízAGA, Leonardo (2005), "La política del estado ecuatoriano hacia los emigrantes nacionales», en Javier Ponce Leiva (ed.), Emigración y política exterior en Ecuador, Quito, Ediciones Abya-Yala.

Banco Central del Ecuador (BCE) (2013), «Evolución de la balanza comercial, enerodiciembre 2012», febrero, en http://www.bce.fin.ec/documentos/Estadisticas/ SectorExterno/BalanzaPagos/balanzaComercial/ebc201302.pdf (04/03/2013). (2012), «Reporte trimestral de mercado laboral», diciembre, en http:// www.bce.fin.ec/documentos/Estadisticas/SectorReal/Previsiones/IndCoyuntura/Empleo/imle201212.pdf (04/03/2013).

(2012), "Reporte de pobreza, desigualdad y mercado laboral», diciembre, en http://www.bce.fin.ec/documentos/Estadisticas/SectorReal/Previsiones/ IndCoyuntura/Empleo/PobrezaDic2012.pdf (04/03/2013).

(2012), «Estadísticas macroeconómicas. Presentación coyuntural», diciembre, en http://www.bce.fin.ec/documentos/Estadisticas/SectorReal/Previsiones/IndCoyuntura/EstMacro122012.pdf (04/03/2013).

Benavides Llerena, Gina (2008), «Políticas migratorias: De la gestión instrumental/ asistencial al reconocimiento de las personas y las garantías de sus derechos», Diálogos Migrantes, núm.1.

Brandi, Maria Carolina (2006), "La historia del brain drain», Revista Iberoamericana de Ciencia, Tecnología y Sociedad, vol. 3, núm.7.

Camacho, Gloria y Hernández Kattya (2005), Cambió mi vida. Migración femenina. Percepciones e impactos, Quito, Unifem, Región Andina y Ceplaes.

CARLING, Jørgen (2007), «Interrogar a las remesas: preguntas centrales para reflexiones más profundas y políticas más adecuadas», en Castles Stephen y Delgado Wise, Raúl (coords.), Migración y desarrollo: Perspectivas desde el Sur, México, Miguel Ángel Porrúa, Universidad Autónoma de Zacatecas.

$128 \frac{\text { SEGUNDO SEMESTRE } 2013}{\text { MIGRACIÓN Y DESARROLLO NÚM. } 21}$ 
CASTLES, Stephen y Miller, Mark (2004), La era de la migración: movimientos internacionales de población en el mundo moderno, México, Miguel Ángel Porrúa, Universidad Autónoma de Zacatecas.

y Delgado Wise Raúl (coords.) (2007), Migración y desarrollo: Perspectivas desde el Sur, México, Miguel Ángel Porrúa, Universidad Autónoma de Zacatecas.

Constitución de la República del Ecuador (2008), en http://www.asambleanacional. gov.ec/documentos/constitucion_de_bolsillo.pdf (27/01/2013).

Correa Delgado, Rafael (2007), Discurso de Posesión, en http://www.efemerides. ec/1/enero/0115_4.htm (12/03/2013).

CourTis, Corina (2011), "Marcos institucionales, normativos y de políticas sobre migración internacional en Argentina, Chile y Ecuador», en Martínez Pizarro Jorge (ed.), Migración Internacional en América latina y el Caribe. Nuevas tendencias, nuevos enfoques, Santiago de Chile, CEPAL.

De la VeGA, Pabloy Túpac-Yupanqui Luis (2004), «Elementos para una política migratoria», en Hidalgo Francisco (ed.), Migraciones. Un juego de cartas marcadas, Quito, Abya Yala, ILDIS-Fes.

García Zamora, Rodolfo (2012), Crisis, migración y desarrollo. Los actores sociales y el reto de las nuevas políticas públicas en México, Zacatecas, Universidad Autónoma de Zacatecas.

(2010), «Migración internacional y desarrollo en América Latina y el Caribe: el mito y la realidad», en América Latina: Democracia, economía y desarrollo social, Madrid, Trama Editorial.

Gómez Ciriano, José Emilio, Tornos Cubillo Andrés y Colectivo ioé (2007), Ecuatorianos en España. Una aproximación sociológica. Observatorio Permanente de la Inmigración, Ministerio de Trabajo y Asuntos Sociales, Subdirección General de Información Administrativa y Publicaciones, Madrid.

Herrera, Gioconda, María Cristina Carrillo y Alicia Torres (eds.) (2005), La migración ecuatoriana. Transnacionalismo, redes e identidades, Quito, Flacso-Sede Ecuador/ Plan Migración Comunicación y Desarrollo.

(coord.) (2008), Ecuador: la migración internacional en cifras, Quito, FlacsoSede Ecuador/Fondo de Población de las Naciones Unidas (UnfPA).

y Moncayo María Isabel (2011),El Plan «Bienvenid@s a casa».Estudio sobre la experiencia del Fondo "El Cucayo», en http://www.fundacioncarolina.es/es-ES/ publicaciones/avancesinvestigacion/Documents/AI51.pdf (30/09/2012). 
Hidalgo, Francisco (ed.) (2004), Migraciones. Un juego de cartas marcadas, Quito, Abya Yala, ILDIS-Fes.

ILDIS (2004), "Características y diferencias clave entre las primeras migraciones y la reciente ola emigratoria», Cartilla sobre Migración, núm. 10. Plan Migración, Comunicación y Desarrollo, Quito, ILDIS-FES.

Instituto Ecuatoriano de Cooperación Internacional (INECI) (2007), «Experiencias y resultados de los procesos de conversión de deuda del Ecuador con España y otros países», en http://segib.org/social/files/2010/09/resultados.pdf (23/10/2012).

JoкIsch, Brad (2007), Ecuador: diversidad en migración. Migration Information Source, en http://www.migrationinformation.org/Profiles/display.cfm?id=591 $(15 / 08 / 2012)$.

Mármora, Lelio (2002), Las políticas de migraciones internacionales, Buenos Aires, Paidós. Ministerio de Coordinación de la Producción, Empleo y Competitividad (2012), "Rendición de cuentas, 2012. Sector producción», en http://www.produccion. gob.ec/rendicion-de-cuentas-2012-consejo-sectorial-de-la-produccion/ (04/03/2013).

(2011), "Agenda para la transformación productiva», en http://www.produccion.gob.ec/wp-content/uploads/downloads/2012/07/Agenda_Productiva[1].pdf (04/03/2013).

OIM (2008), «Perfil Migratorio del Ecuador», en http://publications.iom.int/bookstore/free/ecuador_profile.pdf. (15/08/2012).

(2011), «Perfil Migratorio del Ecuador», en http://publications.iom.int/ bookstore/free/ecuador_profile.pdf. (15/02/2013).

Organización Internacional del Trabajo (OIT) (2013), Panorama laboral 2012. América Latina y el Caribe, Lima.

Palazuelos, Antonio (2013), «Elementos de fortaleza y debilidad del Proyecto de Desarrollo de la Revolución Ciudadana», en Sembrando el desarrollo en América Latina, México, Instituto de Investigaciones Económicas de la Universidad Nacional Autónoma de México.

y Villarreal María del Carmen (2012), «Ecuador: el Proyecto de Desarrollo de la Revolución Ciudadana», en Antonio Palazuelos, José Déniz y Omar De León (coords.), Nuevas Estrategias económicas en América Latina, Madrid, La Catarata.

$130 \frac{\text { SEGUNDO SEMESTRE } 2013}{\text { MIGRACIÓN Y DESARROLLO NÚM. } 21}$ 
Pellegrino, Adela y Calvo Juan José (2001), «żDrenaje o éxodo? Reflexiones sobre la migración calificada», en http://www.universidadur.edu.uy/bibliotecas/trabajos_rectorado/doc_tr12.pdf. (28/11/2012).

y Vigorito, Andrea (2009), «La emigración calificada desde América latina y las iniciativas nacionales de vinculación. Un análisis del caso uruguayo», Revista Pensamiento Iberoamericano, núm. 4.

Ponce Leiva, Javier (ed.) (2005), Emigración y política exterior en Ecuador, Quito, Ediciones Abya Yala.

Portes, Alejandro (2011), «Migración y desarrollo: un intento de conciliar perspectivas opuestas", Nueva Sociedad, núm. 233.

(2007), «Migración y desarrollo: una revisión conceptual de la evidencia», en Castles, Stephen y Delgado Wise Raúl (coords.), Migración y Desarrollo: perspectivas desde el sur, México, Miguel Ángel Porrúa, Universidad Autónoma de Zacatecas.

Postelnicu, Andrei (2010), «Diásporas y desarrollo: un estudio sobre el rol de los migrantes en su país de origen a través de la revisión de la literatura», Revista Relaciones Internacionales, núm. 14.

Prometeo (2013), «Programa Prometeo», en http://prometeo.educacionsuperior.gob. ec/Prometeo/inicio.do (04/11/2012).

Quiloango Tipanluisa, Susana (2011), Políticas públicas migratorias en el Ecuador. Friedrich Ebert Stiftung, ILDIs, en http://library.fes.de/library/fr-digbib-en.html. (30/05/2012).

Ramírez Gallegos, Franklin y Ramírez, Jacques Paul (eds.) (2005), La estampida migratoria ecuatoriana: crisis, redes transnacionales y repertorios de acción migratoria, Quito, Centro de Investigaciones CIUDAD, Abya Yala.

Senami (2013a), «Política Migratoria para el Buen Vivir», en http://www.migrante. gob.ec/politica-migratoria-para-el-buen-vivir/ (06/04/2013).

(2013b), «Boletín no. 93», en http://www.migrante.gob.ec/wp-content/ uploads/downloads/2013/01/BOLET\%C3\%8DN-N\%C2\%BA-93.Incentivosecon\%C3\%B3micos-para-negocios-benefician-a-11.870-personas-migrantes. pdf (06/04/2013).

(2007), «Plan Nacional de Desarrollo Humano para las Migraciones 20072010» en http://www.senami.gov.ec/index2.php?option=com_docman\&task $=$ doc_view\&gid $=108 \&$ Itemid $=98(12 / 07 / 2012)$. 
SENESCYT (2013), «Bases de postulación. Programa de Becas», en http://www2.ucsg. edu.ec/dmdocuments/SENESCYT_Convocatoria.2013.pdf

Senplades (2009), «Plan nacional para el Buen Vivir 2009-2013», en http://www. planificacion.gob.ec/plan-nacional-para-el-buen-vivir-2009-2013/ (09/04/2013).

Solimano, Andrés (2008), Las Migraciones internacionales en América Latina: booms, crisis y desarrollo, Santiago de Chile, Fondo de Cultura Económica.

Yachay (2013), «خ̇Qué es Yachay?», en http://www.yachay.ec/ (04/11/2012). 